\title{
Clostridium Difficile Infection in the "Oldest" Old: Clinical Outcomes in Patients Aged 80 and Older
}

\author{
Eric D. Cober, $M D,{ }^{*}$ and Preeti N. Malani, $M D^{*}+\mathcal{S}$
}

OBJECTIVES: Clostridium difficile infection (CDI) represents a cause of substantial morbidity, particularly for older adults. Although older age is a risk factor for CDI, few studies have specifically focused on clinical outcomes in older adults, particularly the "oldest" old.

DESIGN: Retrospective review.

SETTING: University of Michigan Health System.

PARTICIPANTS: All patients aged 80 and older with a positive cytotoxin assay for $C$. difficile and a clinical course consistent with CDI during 2006.

MEASUREMENTS: Clinical data were recorded, including comorbid conditions and treatment regimens, as well as outcomes, including treatment failure, infection relapse, and 90-day mortality.

RESULTS: Seventy patients aged 80 and older (mean $84.0 \pm 4.1$ ) with CDI were identified. Metronidazole was given as initial therapy in $65(92.8 \%) ; 18$ of these 65 $(27.7 \%)$ experienced treatment failure, requiring subsequent use of oral vancomycin. Serious adverse events included three episodes of toxic megacolon, two requiring colectomy. One death was directly attributable to CDI. Allcause mortality was $8.6 \%$ at 30 days and $17.1 \%$ at 90 days. Higher white blood cell (WBC) counts were independently associated with treatment failure $(P=.02)$ and coronary artery disease with 90 -day mortality $(P=.02)$.

CONCLUSION: In older adults with CDI, treatment failure on metronidazole occurred frequently and was associated with higher WBC count. Larger prospective studies are needed to determine risk factors for treatment failure and relapse in order to develop better paradigms for CDI treatment in older adults. Initial therapy with vancomycin may be appropriate for elderly patients, especially those with elevated WBC counts. J Am Geriatr Soc 57:659-662, 2009.

From the Divisions of * Infectious Diseases and ${ }^{\dagger}$ Geriatric Medicine, Department of Internal Medicine, University of Michigan Health System, Ann Arbor, Michigan; ${ }^{\ddagger}$ Veterans Affairs Ann Arbor Healthcare System, Ann Arbor, Michigan; and ${ }^{\S}$ Geriatric Research Education and Clinical Center, Ann Arbor, Michigan.

Address correspondence to Preeti N. Malani, VA Healthcare System, 2215 Fuller Road, Ann Arbor, MI 48105. E-mail: pmalani@umich.edu

DOI: 10.1111/j.1532-5415.2009.02182.x
Key words: Clostridium difficile; aging; metronidazole; vancomycin

$\mathbf{I}^{\mathrm{n}}$ n recent years, the incidence and severity of Clostridium difficile infection (CDI) have increased. With the emergence of the highly toxigenic strain, BI/NAP1, CDI has emerged as a cause of substantial morbidity and healthcare costs, especially in older adults. ${ }^{1-3}$ In recent reports of BI/ NAP1 outbreaks, age of 65 and older has been highlighted as an important risk factor for developing CDI, as well as for greater disease severity and higher mortality. ${ }^{4-6}$

The Centers for Disease Control and Prevention (CDC) reports that hospital discharge diagnosis of CDI nearly doubled from 1996 to 2003, increasing from 31 to 61 discharges per 100,000 population. ${ }^{5}$ This increase was most notable in older adults; in persons aged 65 and older, rates increased from approximately 150 discharges per 100,000 population in 1996 to 300 discharges per 100,000 population in 2003. In addition to greater incidence and mortality, older adults experience higher rates of treatment failures and disease relapse. ${ }^{5,6}$

Although older age has been repeatedly identified as a risk factor for CDI, few studies have specifically focused on clinical outcomes of CDI in older adults. It was desired to examine the clinical course of CDI in the "oldest" old $(\geq 80)$, including the rate of important complications, such as need for hospitalization or surgical intervention, and death. Treatment failure and relapse of disease within 90 days of initial treatment were also recorded.

\section{METHODS}

\section{Setting and Population}

The University of Michigan Health System (UMHS) is a 850-bed tertiary care facility. Using microbiology records, all patients aged 80 and older cared for at the UMHS with a positive enzyme-linked immunosorbent assay for C. difficile cytotoxin A or B and a clinical course consistent with CDI from January 1, 2006, to December 31, 2006, were identified. Medical records were reviewed and information of 
interest recorded including demographic data, clinical conditions, and characteristics of CDI. We assessed treatment regimens, complications (including toxic megacolon and need for surgery), pre- and post-hospitalization care requirements, and mortality.

\section{Outcomes of Interest}

Specific outcomes of interest were defined as follows failure of initial agent, defined as lack of clinical improvement after 5 days or change in treatment regimen because of lack of clinical response; relapse, defined as any recurrence of CDI within a 90-day period after initial presentation; and death within 90 days of presentation (all-cause mortality). The institutional review boards of the University of Michigan Health System approved this study.

\section{Data Analysis}

Univariate analysis using $t$-tests for continuous variables and the chi-square or Fisher exact test for categorical variables identified risk factors for treatment failure, relapse, and death in patients with CDI. A two-tailed $P \leq .05$ was considered statistically significant. Crude odds ratios (ORs) and $95 \%$ confidence intervals (CIs) were calculated for categorical variables. Crude ORs for continuous variables were obtained using simple logistic regression. Variables that were significant to $P=.20$ and variables that had a priori clinical significance were then analyzed using multivariate logistic regression modeling. All statistical analysis was performed using SAS 9.1 (SAS Institute, Inc., Cary, NC).

\section{RESULTS}

Seventy patient aged 80 and older were identified with CDI (mean age $84.0 \pm 4.1$, range $80-94)$. Twenty-nine (41.1\%) were male. Common medical comorbidities were coronary artery disease $(35.7 \%)$, renal insufficiency $(27.1 \%)$, and diabetes mellitus $(18.5 \%)$. The mean peak white blood cell (WBC) count was $14.8 \pm 8.6$ thousand $/ \mathrm{mm}^{3}$ (range 3.646.4). Fifty-seven $(81.4 \%)$ patients received antibiotics, and $41(58.5 \%)$ received proton pump inhibitors during the 30 days before presentation (Table 1).

Initial therapy for CDI consisted of metronidazole in 65 $(92.8 \%)$, and vancomycin in two $(2.9 \%)$. Three patients did not receive antimicrobial therapy for CDI treatment. Complications associated with CDI included toxic megacolon in three patients $(4.3 \%)$, two of whom required colectomy.

Treatment failure prompting a change in CDI therapy occurred in 18 of 65 patients $(27.7 \%)$, all of whom received initial treatment with metronidazole. All 18 were switched to oral vancomycin. These 18 patients received a median of 7 days of metronidazole before the switch to vancomycin. All 18 had appropriate dosing of metronidazole, generally $500 \mathrm{mg}$ three or four times a day.

Twelve patients $(17.1 \%)$ experienced disease relapse within 90 days after completion of treatment for CDI. All 12 received initial treatment with metronidazole: four with relapsed disease had experienced treatment failure during their initial presentation, prompting a switch to oral vancomycin.
Table 1. Characteristics for Patients Aged 80 and Older with Clostridium Difficile-Associated Diarrhea $(\mathbf{N}=70)$

\begin{tabular}{lc}
\hline \multicolumn{1}{c}{ Characteristic } & Value \\
\hline Age, mean $\pm \mathrm{SE}$ & $84.0 \pm 4.1$ \\
Male, $\mathrm{n}(\%)$ & $29(41.1)$ \\
Coronary artery disease, $\mathrm{n}(\%)$ & $25(35.7)$ \\
Congestive heart failure, $\mathrm{n}(\%)$ & $22(31.4)$ \\
Diabetes mellitus, $\mathrm{n}(\%)$ & $13(18.5)$ \\
Renal insufficiency (creatinine clearance $<30 \mathrm{~mL} / \mathrm{min}), \mathrm{n}(\%)$ & $19(27.1)$ \\
Colon cancer, $\mathrm{n}(\%)$ & $2(2.8)$ \\
Inflammatory bowel disease, $\mathrm{n}(\%)$ & $2(2.8)$ \\
Peak white blood cell count, $\mathrm{K} / \mathrm{mm}^{3}$, mean $\pm \mathrm{SE}$ & $14.8 \pm 8.6$ \\
Surgery during previous month, $\mathrm{n}(\%)$ & $11(15.7)$ \\
Antimicrobial use in past 30 days, $\mathrm{n}(\%)$ & $57(81.4)$ \\
Proton pump Inhibitor in past 30 days, $\mathrm{n}(\%)$ & $41(58.5)$ \\
Initial therapy, $\mathrm{n}(\%)^{*}$ & \\
\hline Metronidazole & $65(93)$ \\
\hline Vancomycin & $2(2.9)$ \\
Complications, $\mathrm{n}(\%)$ & \\
\hline Toxic megacolon & $3(4.3)$ \\
\hline Need for colectomy & $2(2.9)$ \\
Relapse & $12(17.1)$ \\
\hline Treatment failure & $18(25.7)$ \\
\hline Death (within 90 days) & $12(17.1)$ \\
\hline
\end{tabular}

*Three patients did not receive antimicrobial therapy for Clostridium difficile-associated diarrhea.

$\mathrm{SE}=$ standard error.

Twelve patients (17.1\%) died within 90 days of initial presentation and diagnosis; one death was directly attributable to CDI. These deaths occurred from 2 to 85 days after the initial diagnosis of CDI (median 33.5 days). Overall, six deaths occurred within 30 days of presentation $(8.6 \%)$. Neither treatment failure nor disease relapse appeared to be associated with a higher death rate when compared with each other. The death rate for patients experiencing treatment failure was $11.1 \%$; for patients with CDI relapse, it was $8.3 \%$.

Of the 70 patients studied, 31 required 40 admissions with a primary admitting diagnosis of CDI, 15 were treated as outpatients, and 24 developed CDI during hospitalization for another medical or surgical issue. Of the 31 patients admitted for CDI, 25 were community dwelling and independent with activities of daily living before admission; eight of these $25(32.0 \%)$ required discharge to subacute settings because of impaired mobility and functional decline.

Overall, treatment failure occurred in $18(25.7 \%)$ patients. Of the 31 patients admitted primarily for CDI, 11 $(35.5 \%)$ experienced treatment failure. Fifteen patients were treated on an outpatient basis, with three treatment failures $(20 \%)$. Of the 24 patients who developed CDI during an admission for another reason, four experienced treatment failure $(16.6 \%)$. Although the patients admitted with a primary diagnosis of CDI had a higher rate of treatment failure, this difference did not achieve statistical significance $(\mathrm{OR}=2.5,95 \% \mathrm{CI}=0.84-7.6, P=.16)$.

Univariate analysis identified risk factors associated with outcomes of interest (Table 2). Patients experiencing 
treatment failure had a higher peak WBC count than those who responded to initial therapy $(P<.01)$. Coronary artery disease was highly associated with 90 -day mortality (crude $\mathrm{OR}=4.4, P=.05$ ). These risk factors remained independently associated with treatment failure and 90-day mortality on multivariate analysis (Table 3 ).

\section{DISCUSSION}

CDI is a serious illness associated with significant morbidity and mortality. In recent years, the incidence of CDI infection has more than doubled. ${ }^{4}$ Past investigations have noted that older adults who develop CDI are at high risk of serious adverse effects, including death. It was desired to describe the clinical course of patients aged 80 and older, including identification of possible risk factors for poor outcomes and treatment failure.

These findings suggest that, in the "oldest" elderly patients with CDI, treatment failure on metronidazole occurred frequently $(27.7 \%)$ and appears to be associated with higher peak $\mathrm{WBC}$ count $(P=.01)$. The relationship between high WBC count and greater severity of clinical disease is well described; this association was also observed in the initial reports of B1/NAP1 strain outbreaks. ${ }^{2,6}$ The nearly $28 \%$ rate of metronidazole failure observed in this cohort is consistent with the rate of treatment failure in patients with severe CDI described in two recent prospective studies of CDI treatment. ${ }^{7,8}$

Of previously community-dwelling patients admitted with a primary diagnosis of CDI, $32.0 \%$ were discharged to subacute care. Overall death rates were significant, with $17.1 \%$ dying within 90 days of CDI diagnosis and one death directly attributable to CDI. If only hospitalized patients are considered $(\mathrm{n}=55)$, the death rate climbs to $21.8 \%$. Adjusted odds of death in patients with coronary artery disease were more than five times as high as patients without coronary artery disease, $(P=.02)$.

Limitations of this study include the retrospective design and the small sample size. The overwhelming use of metronidazole as the first line of therapy in these patients makes it impossible to compare the efficacy of metronidazole with that of oral vancomycin. Despite these limitations,

Table 3. Multivariate Analysis of the Association Between Patient Characteristics, Treatment Failure, and 90-Day Mortality in Patients with Clostridium Difficile-Associated Diarrhea

\begin{tabular}{llc}
\hline & \multicolumn{1}{c}{$\begin{array}{c}\text { Odds Ratio (95\% Confidence } \\
\text { Interval) } \boldsymbol{P} \text {-Value }\end{array}$} \\
\cline { 2 - 3 } & \multicolumn{1}{c}{ Unadjusted } & \multicolumn{1}{c}{ Adjusted* } \\
\hline $\begin{array}{c}\text { Treatment failure } \\
\text { White blood cell count }\end{array}$ & $1.1(1.0-1.2) .007$ & $1.1(1.0-1.2) .02$ \\
90-day mortality & & \\
$\quad$ Coronary artery disease & $4.4(1.2-16.7) .05$ & $5.5(1.3-23) .02$ \\
$\quad$ White blood cell count & $1.0(0.9-1.1) .35$ & $1.1(1.0-1.2) .13$ \\
\hline
\end{tabular}

* Based on a multivariable logistic regression adjusting for age, renal insufficiency, serum albumin, coronary artery disease, congestive heart failure, diabetes mellitus. 
the $27.7 \%$ rate of treatment failure in patients receiving metronidazole is notable and suggests that metronidazole may not be the appropriate initial agent in older adults, particularly those with high WBC counts on presentation or other signs and symptoms of severe CDI. The question of optimal therapy for CDI remains unclear, although it is evolving. Many experts have recommended vancomycin as first-line therapy in older adults, especially for patients with severe disease, characterized by high WBC count, severe abdominal pain, or rising creatinine. ${ }^{7,9-11}$ Several clinical studies have demonstrated that oral vancomycin provides higher rates of cure than metronidazole in patients with severe CDI, ${ }^{7,8}$ although drug costs remain a major barrier for use of vancomycin as a first line of therapy, particularly in mild or moderate disease, where there is no evidence that vancomycin is superior to treatment with metronidazole. ${ }^{12}$

As the burden of CDI in older adults continues to grow, so does the need to improve understanding of disease in this population. Larger prospective studies are needed to determine risk factors for CDI treatment failure, relapse, and poor outcomes in order to develop better paradigms for CDI treatment for older adults, including how to identify patients who should receive initial therapy with oral vancomycin and patients who may benefit from extended treatment courses or tapered therapy. Efforts to prevent CDI through directed infection control measures and prudent use of antimicrobials remain critical, particularly in the long-term and subacute care settings. Finally, the problem of functional decline associated with the diagnosis of CDI deserves consideration. Although pre- and postadmission functional ability was not formally assessed, a sizable number of patients previously independent in activities of daily living admitted specifically for CDI required subacute care after discharge.

\section{ACKNOWLEDGMENTS}

This work was presented in part at the 45th Annual Scientific Meeting of the Infectious Diseases Society of America, San Diego, California, October 4 to 7, 2007 (Poster 977).

Conflict of Interest: All authors declare that they have no conflicts of interest.
This work was supported in part by the Veterans Affairs Ann Arbor Healthcare System, Geriatric Research, Education and Clinical Center; John A. Hartford Foundation's Center of Excellence; and an unrestricted educational grant from ViroPharma Incorporated.

Author Contributions: Cober: data collection, manuscript preparation. Malani: study design, data collection, analysis and interpretation of data, manuscript preparation.

Sponsor's Role: None.

\section{REFERENCES}

1. Loo V, Poirier L, Miller M et al. A predominantly clonal multi-institutional outbreak of Clostridium difficile-associated diarrhea diarrhea with high morbidity and mortality. N Engl J Med 2005;353:2442-2449.

2. McEllistrem M, Carman R, Gerding D et al. A hospital outbreak of Clostridium difficile disease associated with isolates carrying binary toxin genes. Clin Infect Dis 2005;40:265-272.

3. Muto CA, Pokrywka M, Shutt K et al. A large outbreak of Clostridium difficile-associated disease with an unexpected proportion of deaths and colectomies at a teaching hospital following increased fluoroquinolone use. Infect Control Hosp Epidemiol 2005;26:273-280.

4. McDonald L, Owings M, Jernigan D. Clostridium difficile infection in patients discharged from US short-stay hospital, 1996-2003. Emerg Infect Dis 2006;12:409-414

5. Pepin J, Valiquette L, Alary $\mathrm{M}$ et al. Clostridium difficile-associated diarrhea in a region of Quebec from 1991 to 2003: A changing pattern of disease severity. CMAJ 2004;171:466-472.

6. Pepin J, Valiquette L, Cossette B. Mortality attributable to nosocomial Clostridium difficile-associated disease during an epidemic caused by a hypervirulent strain in Quebec. CMAJ 2005;173:1037-1042.

7. Zar FA, Bakkanagari R, Moorthi KMLST et al. A comparison of vancomycin and metronidazole for the treatment of Clostridium difficileassociated diarrhea, stratified by disease severity. Clin Infect Dis 2007;45: 302-307.

8. Louie T. Results of a phase III trial comparing tolevamer, vancomycin and metronidazole in Clostridium difficile-associated diarrhea (CDAD) (abstract K-4259). In: Program and abstracts of the 47th Interscience Conference on Antimicrobial Agents and Chemotherapy (Washington DC). Herndon, VA: ASM Press, 2007.

9. Gerding D. Metronidazole for Clostridium difficile-associated disease: Is it okay for mom? Clin Infect Dis 2005;40:1598-1560.

10. Bartlett JG. The case for vancomycin as the preferred drug for treatment of Clostridium difficile infection. Clin Infect Dis 2008;46:1489-1492.

11. Musher D, Aslam S, Logan $\mathrm{N}$ et al. Relatively poor outcome after treatment of Clostridium difficile colitis with metronidazole. Clin Infect Dis 2005;40:15861590.

12. Pepin J. Vancomycin for the treatment of Clostridium difficile infection: For whom is this expensive bullet really magic? Clin Infect Dis 2008;46: 1493-1498. 